\title{
Oxidative Stress in Cystinosis Patients
}

\author{
Maria Helena Vaisbich ${ }^{\mathrm{a}}$ Luciana Pache de Faria Guimaraes $^{\mathrm{a}}$ \\ Maria Heloisa Mazzola Shimizu ${ }^{b}$ Antonio Carlos Seguro ${ }^{b}$ \\ a Pediatric Nephrology Unit, Instituto da Criança, and b Medical Investigation Laboratory 12, \\ Department of Nephrology, Hospital das Clínicas, Faculdade de Medicina, Universidade de \\ São Paulo, São Paulo, Brazil
}

\section{Key Words}

Biomarkers $\cdot$ Chronic kidney disease $\cdot$ Cysteamine $\cdot$ Cystinosis $\cdot$ End-stage renal disease $\cdot$ Oxidative stress $\cdot$ Thiobarbituric acid

\begin{abstract}
Background/Aims: Nephropathic cystinosis (NC) is a severe systemic disease and cysteamine improves its prognosis. Lysosomal cystine accumulation is the hallmark of cystinosis and is regarded as the primary defect due to mutations in the CTNS gene. However, there is great evidence that cystine accumulation itself is not responsible for all abnormalities observed in NC. Studies have demonstrated altered ATP metabolism, increased apoptosis, and cell oxidation. An increased number of autophagosomes and autophagic vacuoles have been observed in cystinotic fibroblasts and renal epithelial cells, suggesting that altered autophagy plays a role in $\mathrm{NC}$, leading to increased production of reactive oxygen species. Therefore, cystinosis patients can be more susceptible to oxidative stress (OS) and it can contribute to the progression of the renal disease. Our goal was to evaluate a marker of OS (serum TBARS) in NC children, and to compare the results with those observed in healthy controls and correlated with renal function parameters. Methods: The study included patients aged under 18 years, with good adherence to the treatment and out of renal replacement therapy. The following parameters were evaluated: serum creatinine, BUN, creatinine clearance estimated by stature and serum TBARS levels. Results: We selected 20 patients aged $8.0 \pm 3.6$ years and observed serum TBARS levels of 4.03 $\pm 1.02 \mathrm{nmol} / \mathrm{ml}$. Serum TBARS levels in the 43 healthy controls, aged $7.4 \pm 1.1$ years, were 1.60 $\pm 0.04 \mathrm{nmol} / \mathrm{ml}$. There was a significant difference between the plasma TBARS levels among the 2 groups $(p<0.0001)$. We detected no significant correlation between plasma TBARS levels and renal function. Conclusion: An increased level of serum TBARS in patients with NC was observed and this abnormality was not correlated with the renal function status degree. This is the first report that shows increased oxidative stress in serum of NC patients.
\end{abstract}

Copyright $\odot 2011$ S. Karger AG, Basel 


\section{Introduction}

Nephropathic cystinosis is a severe systemic progressive disease. It involves several organs throughout life. First, the kidneys are affected by proximal tubular dysfunction and Fanconi's syndrome. During childhood, there is also frequent hypothyroidism and ocular involvement, leading to photophobia. Renal glomerular damage generally becomes apparent between 2 and 5 years of age and results in end-stage renal disease between 9 and 10 years of age, unless treatment with a cystine-depleting drug, cysteamine, is initiated early in life [1]. Patients without this treatment, besides presenting glomerular damage and chronic renal insufficiency, can also present pancreatic problems, e.g. diabetes mellitus and high levels of amylase, hepatic involvement, gastrointestinal complications, e.g. vomiting or gastric ulcers, muscular wasting and weakness, and various degrees of central nervous system involvement $[1,2]$. However, although lysosomal cystine accumulation is the hallmark of cystinosis and is regarded as the primary defect due to mutations in the CTNS gene, there is great evidence that cystine accumulation itself is not responsible for all abnormalities observed in cystinosis. Several studies have recently demonstrated altered ATP metabolism, increased apoptosis, and cell oxidation. For the most part, these abnormalities were observed using cell models and are not necessarily mutually exclusive [3]. Moreover, an increased number of autophagosomes and autophagic vacuoles have been observed in cystinotic fibroblasts and renal epithelial cells, suggesting that altered autophagy also plays a role in cystinosis [4]. These observations were associated with structural mitochondrial abnormalities and increased production of reactive oxygen species (ROS). Taken together, these findings suggest that cystinosis patients can be more susceptible to oxidative stress, which can contribute to the progression of renal disease in cystinosis patients even when they show a good response to treatment with cysteamine $[5,6]$.

Our aim was to evaluate a marker of oxidative stress in pediatric cystinosis and compare the results obtained in cystinotic children with those observed in healthy normal controls. We also correlated the oxidative stress marker with renal function parameters in these patients. Of note, this is the first documentation of increased oxidative stress in the serum of cystinotic patients.

\section{Patients and Methods}

\section{Patients}

The study cohort was selected from cystinosis patients aged $<18$ years. Inclusion criteria were parental consent for participation in the study and good adherence to the treatment, including their attendance at the follow-up examinations and clinical evaluations, and no renal replacement therapy. Only patients with chronic kidney disease (CKD) in stage 1-4 (based on KDOQI guidelines [7]) were included.

All patients selected had taken cysteamine at a recommended dosage of $60-90 \mathrm{mg} / \mathrm{kg} /$ day [5]. Sample collections to measure the parameters of the study were done at the time when patients were under metabolic and electrolyte control, and free of infections.

\section{Methods}

The following renal function parameters were evaluated: serum creatinine, BUN and creatinine clearance estimated by the Schwartz formula [8].

Serum levels of thiobarbituric acid-reactive substances (TBARS), which are markers of lipid peroxidation, were determined using the TBARS assay. In brief, $0.2 \mathrm{ml}$ of serum sample was diluted in $0.8 \mathrm{ml}$ of distilled water. Immediately thereafter, $1 \mathrm{ml}$ of $17.5 \%$ trichloro- 
Table 1. Cystinosis patients $(n=20)$ according to CKD stage

$\begin{array}{ll}\text { CKD stage } 1 & 9 \text { patients } \\ \text { CKD stage } 2 & 5 \text { patients } \\ \text { CKD stage } 3 & 5 \text { patients } \\ \text { CKD stage } 4 & 1 \text { patient }\end{array}$

Table 2. Parameters measured in the 20 cystinosis patients

$\begin{array}{ll}\text { Serum creatinine, } \mu \mathrm{mol} / \mathrm{l} & \\ \quad \text { Median } & 59.5 \\ \text { Interquartile range } & 39.6-93.0 \\ \text { Geometric mean, log transformed } & 61.0 \\ \text { BUN, mmol/l } & 10.3 \pm 5.7 \\ \text { eGFR (Schwartz formula), } \mathrm{ml} / \mathrm{min} / 1.73 \mathrm{~m}^{2} & 84.0 \pm 41.5 \\ \text { Serum TBARS, nmol/ml } & 4.03 \pm 1.02\end{array}$

acetic acid was added. Following the addition of $1 \mathrm{ml}$ of $0.6 \%$ thiobarbituric acid, $\mathrm{pH} 2$, the sample was placed in a boiling water bath for $15 \mathrm{~min}$, after which it was allowed to cool. Subsequently, $1 \mathrm{ml}$ of $70 \%$ trichloroacetic acid was added, and the mixture was incubated for $20 \mathrm{~min}$. The sample was then centrifuged for $15 \mathrm{~min}$ at 2,000 r.p.m. The optical density of the supernatant was read at $534 \mathrm{~nm}$ against a reagent blank using a spectrophotometer. The quantity of TBARS was calculated using a molar extinction coefficient of $1.56 \times 10^{5}$ $\mathrm{M}^{-1} \mathrm{~cm}^{-1}$.

Serum levels of TBARS (expressed as $\mathrm{nmol} / \mathrm{ml}$ [9]) were compared between patients and healthy controls, and associations between this parameter and parameters of renal function were assessed.

\section{Statistical Analysis}

Homogenous data are expressed as means \pm SD, and non-homogenous data are expressed as medians (interquartile ranges). The independent sample $t$ test was employed to compare serum levels of TBARS between the patients and healthy control children, and Pearson's correlation coefficient was used to correlate serum TBARS with renal function parameters. This study was approved by the local ethics committee. Informed consent was obtained from the parents of all children for participation in this study.

\section{Results}

We selected 20 cystinosis patients (6 females) aged $8.0 \pm 3.6$ years (mean \pm SD) in CKD stage 1-4 with good adherence to the treatment comprising cysteamine. The distribution of the patients according to CKD stage is shown in table 1 . The dosage of cysteamine was 72.06 $\pm 5.22 \mathrm{mg} / \mathrm{kg} /$ day and was divided every $6 \mathrm{~h}$.

Table 2 shows the results found in these cystinosis patients. In the 43 healthy controls aged $7.4 \pm 1.1$ years (mean \pm SD), serum levels of TBARS were $1.60 \pm 0.04 \mathrm{nmol} / \mathrm{ml}$ (mean $\pm \mathrm{SD}$ ); in cystinosis patients, serum TBARS levels were $4.03 \pm 1.02 \mathrm{nmol} / \mathrm{ml}$ (mean $\pm \mathrm{SD}$ ). There was no statistical difference between patients and healthy controls regarding age, but 

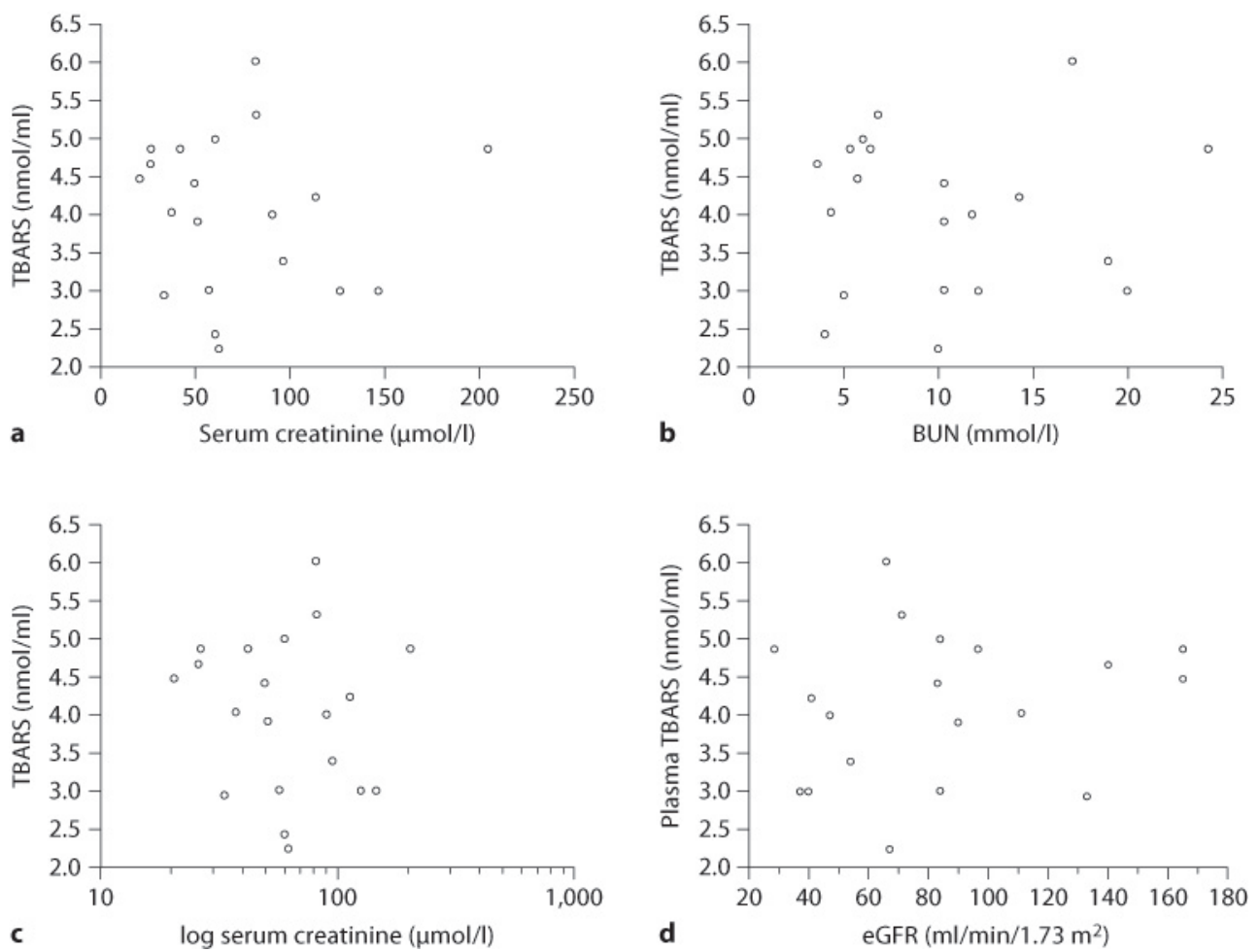

Fig. 1. Correlation between plasma TBARS and serum creatinine (a, c), BUN (b) and creatinine clearance estimated by stature [eGFR (Schwartz formula); d] in nephropathic cystinosis patients. a $\mathrm{r}=-0.04, \mathrm{p}=$ 0.86 . b $\mathrm{r}=0.02, \mathrm{p}=0.93$. $\mathrm{c} \mathrm{r}=-0.10, \mathrm{p}=0.65 . \mathrm{d} \mathrm{r}=0.17, \mathrm{p}=0.46$

there was a significant difference between the plasma TBARS levels between both groups $(\mathrm{p}<0.0001)$.

To determine the effect of renal function impairment on serum TBARS, we correlated this parameter with serum creatinine, BUN, creatinine clearance estimated by stature and CKD stage. No significant correlations between plasma TBARS and all these parameters were detected (fig. 1).

\section{Discussion}

Nephropathic cystinosis is a severe disease. Although cysteamine treatment improves its prognosis, renal failure is not completely abolished. Gahl et al. [6] emphasized that cysteamine is not $100 \%$ effective, even taking all appropriate steps, since some patients present extrarenal complications and renal failure at the age of 20 years. Likewise, Greco et al. [10] have also demonstrated that end-stage renal failure cannot be prevented in most patients but is only postponed to the 2 nd or 3 rd decade of life; late-onset complications ultimately develop in a substantial proportion of patients. Ergo, nephropathic cystinosis remains a challenge for the treating physician.

Recently, several studies have demonstrated altered ATP metabolism, and increased apoptosis and cell oxidation in cystinosis [3]. In addition, increased autophagy is involved leading to increased ROS production [4]. Most of the studies have been conducted in cell 
models, but a study assessing oxidative stress in the serum of the patients has not been published to date. Therefore, our aim was to evaluate serum TBARS levels in pediatric cystinosis patients. Using this simple methodology, we observed that plasma TBARS, a biomarker of oxidative stress, was increased in patients with nephropathic cystinosis. The balance between oxidants and antioxidants is considerably distorted in patients with chronic renal insufficiency. Evidence suggests that ROS are not merely the consequence of treatment or progressing disease but also one of the causal agents of chronic renal impairment, and that oxidative stress can occur even in the absence of hemodialysis. Patients with uremia have diminished response to oxidative stress probably due to a decrease in the antioxidant capacity; the mechanisms underlying this decrease, however, remain to be elucidated [11]. However, in this study, we could not correlate TBARS levels with creatinine clearance, CKD stage, serum creatinine or BUN. Therefore, in cystinosis patients oxidative stress could be a result of the intrinsic mechanism of the disease [3]. Oxidative stress results in the formation of highly reactive and unstable lipid hydroperoxides, and decomposition of unstable peroxides derived from polyunsaturated fatty acids results in the formation of malondialdehyde, which is measured by the TBARS assay. However, a drawback of this assay is that there may be other sources of malondialdehyde. Thus, this test is not specific for lipid peroxidation.

The limitations of our study are the small number of patients and the fact that TBARS is not the best oxidative stress marker. Although this is only a pilot study, it is of paramount importance since it is the first report proving that oxidative stress is increased in the serum of patients with cystinosis independent of the state of renal function. Therefore, we intend to assess more specific markers in future studies.

This knowledge may have implications on the treatment of cystinosis patients, since oxidative stress is involved in the progression of renal disease, especially in these patients [11].

\section{References}

1 Gahl WA: Cystinosis coming of age. Adv Paediatr 1986;33:95-126.

2 Gahl WA, Thoene MD, Schneider JA: Cystinosis. N Engl J Med 2002;347:111-120.

3 Wilmer MJ, Emma F, Levtchenko EN: The pathogenesis of cystinosis: mechanisms beyond cystine accumulation. Am J Physiol Renal Physiol 2010;299:F905-F916.

4 Sansanwal P, Yen B, Gahl WA, Ma Y, Ying L, Wong LJ, Sarwal MM: Mitochondrial autophagy promotes cellular injury in nephropathic cystinosis. J Am Soc Nephrol 2010;21:272-283.

5 Vaisbich MH, Koch VH, Brazilian Cystinosis Study Group: Report of a Brazilian multicenter study on nephropathic cystinosis. Nephron Clin Pract 2010;114:c12-c18.

6 Gahl WA, Balog JZ, Kleta R: Nephropathic cystinosis in adults: natural history and effects of oral cysteamine therapy. Ann Intern Med 2007;147:242-250.

7 Hogg RJ, Furth S, Lemley KV, Portman R, Schwartz GJ, Coresh J, Balk E, Lau J, Levin A, Kausz AT, Eknoyan G, Levey AS: National Kidney Foundation's Kidney Disease Outcomes Quality Initiative clinical practice guidelines for chronic kidney disease in children and adolescents: evaluation, classification, and stratification. Pediatrics 2003;111:1416-1421.

8 Schwartz GJ, Haycock GB, Edelmann CM Jr, Spitzer A: A simple estimate of glomerular filtration rate in children derived from body length and plasma creatinine. Pediatrics 1976;58:259-263.

9 Shimizu MHM, Coimbra TM, Araujo M, Menezes LF, Seguro AC: N-acetylcysteine attenuates the progression of chronic renal failure. Kidney Int 2005;68:2208-2217.

10 Greco M, Brugnara M, Zaffanello M, Taranta A, Pastore A, Emma F: Long-term outcome of nephropathic cystinosis: a 20-year single-center experience. Pediatr Nephrol 2010;25:2459-2467.

11 Romeu M, Nogues R, Marcas L, Sánchez-Martos V, Mulero M, Martinez-Vea A, Mallol J, Giralt M: Evaluation of oxidative stress biomarkers in patients with chronic renal failure: a case control study. BMC Res Notes 2010;3:20. 